\title{
ARTICLE OPEN \\ Engineering the quantum states of light in a Kerr-nonlinear resonator by two-photon driving
}

\author{
Shruti Puri ${ }^{1}$, Samuel Boutin ${ }^{1}$ and Alexandre Blais ${ }^{1,2}$
}

Photonic cat states stored in high-Q resonators show great promise for hardware efficient universal quantum computing. We propose an approach to efficiently prepare such cat states in a Kerr-nonlinear resonator by the use of a two-photon drive. Significantly, we show that this preparation is robust against single-photon loss. An outcome of this observation is that a twophoton drive can eliminate undesirable phase evolution induced by a Kerr nonlinearity. By exploiting the concept of transitionless quantum driving, we moreover demonstrate how non-adiabatic initialization of cat states is possible. Finally, we present a universal set of quantum logical gates that can be performed on the engineered eigenspace of such a two-photon driven resonator and discuss a possible realization using superconducting circuits. The robustness of the engineered subspace to higher-order circuit nonlinearities makes this implementation favorable for scalable quantum computation.

npj Quantum Information (2017)3:18; doi:10.1038/s41534-017-0019-1

\section{INTRODUCTION}

Characterized by photon-photon interaction, Kerr-nonlinear resonators (KNR) display very rich physics and are consequently the focus of much theoretical and experimental work. ${ }^{1}$ These nonlinear oscillators exhibit bifurcation, ${ }^{2}$ can be used to generate squeezed radiation and for quantum limited amplification, ${ }^{3,4}$ and have been proposed as a resource for quantum logic. ${ }^{5}$ Moreover, a KNR initialized in a coherent state evolves to a quantum superposition of out-of-phase coherent states, also known as a cat state. ${ }^{6}$ In practice, Kerr nonlinearities $K$ in atomic systems are, however, often small in comparison to photon loss rate $\kappa_{1}^{7}$ making the observation of these non-classical states of light difficult. As an alternative approach, strong photon-photon interaction can readily be realized in superconducting quantum circuits, with $\mathrm{K} / K \sim 30$ demonstrated experimentally. ${ }^{8}$ This has led to the observation of cat states in the transient dynamics of a KNR realized by coupling a superconducting qubit to a microwave resonator. ${ }^{8}$ These photonic cat states play an important role in understanding the role of decoherence in macroscopic systems, ${ }^{9}$ in precision measurements ${ }^{10}$ and are useful for quantum computation. ${ }^{11,}{ }^{12}$ However, because of their sensitivity to undesirable interactions and photon loss, high-fidelity preparation and manipulation of these states is challenging.

To address this problem, new ideas building on engineered dissipation and taking advantage of the strong nonlinearities that are possible with superconducting circuits have recently been explored theoretically and experimentally. ${ }^{13-18}$ One such approach, known as the qcMAP gate, relies on the strong dispersive qubit-field interaction that is possible in circuit quantum electrodynamics (QED) ${ }^{19}$ to transfer an arbitrary state of a superconducting qubit into a multi-legged cat state. ${ }^{13}, 15,17$ This method is, however, susceptible to single-photon loss that decoheres the cat. This loss also reduces the amplitude of the cat, something that must be compensated for by re-pumping in order to avoid significant overlap between the coherent states. ${ }^{13,}{ }^{15} \mathrm{~A}$ second approach exploits engineered two-photon dissipation realized by coupling a superconducting qubit to two microwave cavities. ${ }^{14,20}$ In the absence of single-photon loss, the steady-state of the field is a cat state whose parity depends on the initial number state of the field. To preserve coherence of the cat, an important experimental challenge is that the rate of single-photon loss must be much smaller than the rate of two-photon loss.

In this paper we propose an experimentally simple alternative approach to encode and stabilize cat states based on two-photon driving of a KNR. This method takes advantage of the fact that the coherent states $| \pm a\rangle$ and, consequently the cat states $\left|\mathcal{C}_{a}^{ \pm}\right\rangle=$ $\mathcal{N}_{a}^{ \pm}(|a\rangle \pm|-a\rangle)$ with $\mathcal{N}_{a}^{ \pm}=1 / \sqrt{2\left(1 \pm e^{-2|a|^{2}}\right)}$, are degenerate eigenstates of the KNR under two-photon driving. Remarkably, this property holds true even in the presence of single-photon loss making this protocol particularly robust and obviating the need for energy re-pumping. Moreover, in contrast to the abovementioned scheme, cat state preparation with this approach does not require dissipation but rather relies on adiabatically turning on the two-photon drive, the number state $|0 / 1\rangle$ evolving into $\left|\mathcal{C}_{a(t)}^{+/-}\right\rangle$. We find that the fidelity of this preparation approaches unity when the Kerr nonlinearity $K$ is large with respect to the photon loss rate $\kappa$, something that is easily realized in current circuit QED experiments. By exploiting the concept of transitionless quantum driving, we show that rapid, non-adiabatic cat state preparation is possible by controlling the amplitude and phase of the two-photon drive. ${ }^{21}$

While large Kerr nonlinearities can be used to produce cat states, it also leads to undesired deformations of these states. ${ }^{6,8}$ This deformation is problematic for qubit-based schemes because of the spurious Kerr nonlinearity inherited by the field from the qubit. $^{22,} 23$ This affects, for example, the qcMAP protocol where

\footnotetext{
${ }^{1}$ Institut quantique and Départment de Physique, Université de Sherbrooke, Sherbrooke, Québec J1K 2R1, Canada and ${ }^{2}$ Canadian Institute for Advanced Research, Toronto, Canada

Correspondence: Shruti Puri (Shruti.Puri@USherbrooke.ca)
}

Received: 2 November 2016 Revised: 6 March 2017 Accepted: 19 March 2017

Published online: 19 April 2017 
the qubit-induced Kerr nonlinearity leads to undesirable phase evolution and distortion of the cat state. Although this deterministic phase evolution can be corrected with qubit-induced-gates, this exposes the field to the decoherence channel of the qubit. ${ }^{24}$ Moreover, in the presence of photons loss, this phase evolution leads to non-deterministic phase errors. ${ }^{18}$ We show how the addition of a two-photon drive of appropriate amplitude and phase during the qcMAP cancels this distortion and the corresponding dephasing.

Taking advantage of the engineered subspace of a two-photon driven KNR, we consider a universal set of gates for an encoding where the coherent states $\{|+a\rangle,|-a\rangle\}$ are mapped to the logical states $\{|\overline{0}\rangle,|\overline{1}\rangle\}$. This mapping is possible because of the quasiorthogonality of coherent states for large $a .^{14}$ We show that highfidelity operations can be realized with realistic parameters. Finally, we discuss realizations based on superconducting Josephson parametric amplifiers which allow the implementation of a twophoton drive along with a Kerr nonlinearity. This simple setup is attractive for building a large scale quantum computing architecture.

\section{RESULTS}

Our starting point is the two-photon driven KNR Hamiltonian in a frame rotating at the resonator frequency

$\hat{H}_{0}=-K \hat{a}^{\dagger} \hat{a}^{\dagger} \hat{a} \hat{a}+\left(\mathcal{E}_{\mathrm{p}} \hat{a}^{\dagger 2}+\mathcal{E}_{\mathrm{p}}^{*} \hat{a}^{2}\right)$.

In the above expression, $K$ is the amplitude of the Kerr nonlinearity and $\mathcal{E}_{\mathrm{p}}$ the amplitude of the two-photon drive. The above Hamiltonian, known as the Cassinian oscillator Hamiltonian, ${ }^{25}$ can be re-written as

$\hat{H}_{0}=-K\left(a^{\dagger 2}-\frac{\mathcal{E}_{\mathrm{p}}^{*}}{K}\right)\left(a^{2}-\frac{\mathcal{E}_{\mathrm{p}}}{K}\right)+\frac{\left|\mathcal{E}_{\mathrm{p}}\right|^{2}}{K}$.

This form of the Hamiltonian illustrates that the two coherent states $| \pm a\rangle$ with $a=\left(\mathcal{E}_{p} / K\right)^{1 / 2}$, which are the eigenstates of the annihilation operator $\hat{a}$, are also degenerate eigenstates of Eq. (1) with energy $\left|\mathcal{E}_{\mathrm{p}}\right|^{2} / K$. Equivalently, the even-odd parity states $\left|\mathcal{C}_{a}^{ \pm}\right\rangle$ are also the eigenstates of $\hat{H}_{0}$. This argument can be generalized to Hamiltonians of the form $-K \hat{a}^{\dagger n} \hat{a}^{n}+\left(\mathcal{E}_{\mathrm{p}} \hat{a}^{\dagger n}+\mathcal{E}_{\mathrm{p}}^{*} \hat{a}^{n}\right)$ that have a set of $n$ coherent states as degenerate eigenstates (see Methods).

In the presence of single-photon loss, the resonator state evolves according to the master equation $\hat{\rho}=-i\left(\hat{H}_{\text {eff }} \hat{\rho}-\hat{\rho} \hat{H}_{\text {eff }}^{\dagger}\right)+$ $\kappa \hat{a} \hat{\rho} \hat{a}^{\dagger}$, with the non-Hermitian effective Hamiltonian $\hat{H}_{\text {eff }}=\hat{H}_{0}-i \kappa \hat{a}^{\dagger} \hat{a} / 2 .^{26}$ While the steady-state of this master equation can be obtained analytically, 27,28 it is simple to show (see Methods) that for $\kappa / 8\left|K a_{0}^{2}\right| \ll 1$ the coherent states $\left| \pm a_{0}\right\rangle=$ $\left| \pm r_{0} e^{i \theta_{0}}\right\rangle$ are degenerate eigenstates of $\hat{H}_{\text {eff }}$ with

$r_{0}=\left(\frac{4 \mathcal{E}_{\mathrm{p}}^{2}-\kappa^{2} / 4}{4 K^{2}}\right)^{1 / 4}, \quad \tan 2 \theta_{0}=\frac{\kappa}{\sqrt{16 \mathcal{E}_{\mathrm{p}}^{2}-\kappa^{2}}}$.

This reduces to the eigenstates of $\hat{H}_{0}$ in the absence of photon loss. The angle $\theta_{0}$ is determined by $\mathcal{E}_{\mathrm{p}}$, with $\theta_{0}<0\left(\theta_{0}>0\right)$ for $\mathcal{E}_{\mathrm{p}}>$ $0\left(\mathcal{E}_{\mathrm{p}}<0\right)$. The last term of the master equation, $\kappa a \hat{\rho} a^{\dagger}$, induces nondeterministic quantum jumps between the even and the odd parity cat states, $\left|\mathcal{C}_{\alpha_{0}}^{+}\right\rangle$and $\left|\mathcal{C}_{a_{0}}^{-}\right\rangle$, leading to decoherence, but not to leakage out of the degenerate subspace $\left\{\left|\mathcal{C}_{a_{0}}^{ \pm}\right\rangle\right\}$. In steadystate, the density matrix therefore takes the form $\hat{\rho}_{\mathrm{s}}=\left(\left|a_{0}\right\rangle\right.$ $\left.\left\langle a_{0}|+|-a_{0}\right\rangle\left\langle-a_{0}\right|\right) / 2^{27,28}$ (see Methods).

Figure 1 shows the steady-state Wigner function for $\kappa / 8\left|k a_{0}^{2}\right| \sim$ $1 / 4$ and $\sim 1 / 16$ obtained by numerical integration of the master equation. ${ }^{29,} 30$ Even for the relatively large value of $k / 8\left|k a_{0}^{2}\right| \sim 1 / 16$ shown in panel a), the steady-state approaches the ideal case $\hat{\rho}_{\mathrm{s}}$ with a fidelity of $99.91 \%$. As expected and evident from Fig. $1 \mathrm{~b}$, the coherent states are deformed at the larger value of $\kappa / 8\left|k a_{0}^{2}\right| \sim$ $1 / 4$ and the fidelity with respect to the ideal steady state is reduced to $96.55 \%$. These numerical results confirm that, even in the presence of single-photon loss, it is possible to confine the

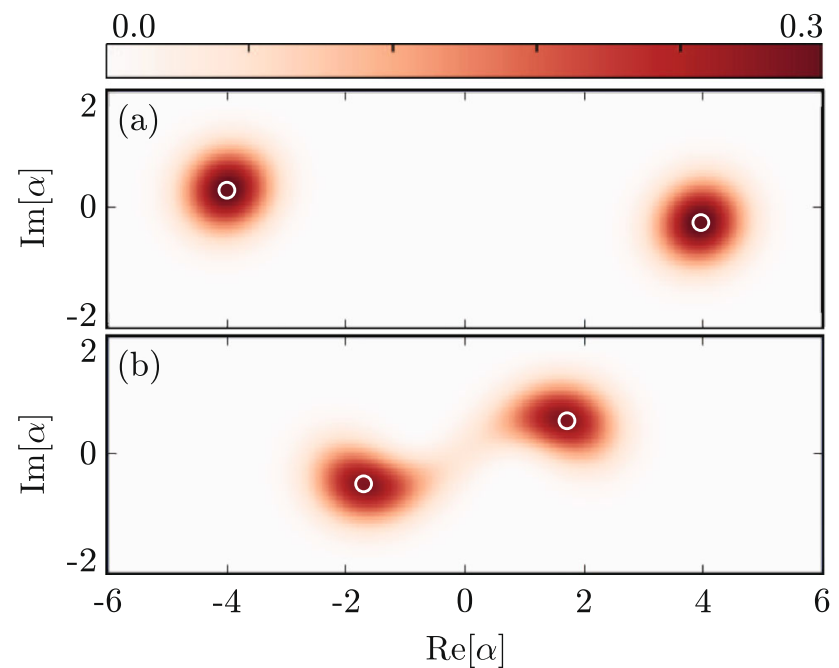

Fig. 1 Steady-state Wigner function of a two-photon driven KNR with $|K| / K=1 / 8$ and a $\mathcal{E}_{p}=16 K, K>0$ and b $\mathcal{E}_{p}=4 K, \quad K<0$, corresponding to $K / 8\left|K a_{0}^{2}\right| \sim 1 / 16$ and $\sim 1 / 4$, respectively. The white circles indicate the expected position of the coherent states following Eq. (3)

state of the resonator to the manifold of coherent states $\left| \pm a_{0}\right\rangle$. Although the photon loss channel remains the dominant source of error, the resonator can also have small amount of dephasing noise, which can cause jumps between $\left|a_{0}\right\rangle$ and $\left|-a_{0}\right\rangle$. With this bit-flip rate decreasing exponentially with $a_{0}{ }^{14}$ (see also Supplementary Information S2), this channel is neglected here.

\section{Adiabatic initialization of cat states}

Going beyond steady-states, we now describe a protocol to deterministically prepare cat states. The vacuum $|n=0\rangle$ and the single-photon Fock state $|n=1\rangle$ are the two-degenerate eigenstates of the undriven KNR. Under the application of a timedependent two-photon drive $\mathcal{E}_{\mathrm{p}}(t)$, the instantaneous eigenstates of the system are the degenerate states $\left| \pm a_{0}(t)\right\rangle$ (or equivalently $\left.\left.\mathcal{C}_{a_{0}(t)}^{ \pm}\right\rangle\right)$, where $a_{0}(t)$ is given by Eq. (3). Since the two-photon drive preserves parity, under adiabatic increase of $\mathcal{E}_{\mathrm{p}}(t)$, the vacuum state $|0\rangle$ evolves to the even parity cat state $\left\langle\mathcal{C}_{\alpha_{0}(t)}^{+}\right\rangle$while the single-photon Fock state evolves to the odd parity cat state $\left.\mathcal{C}_{\alpha_{0}(t)}^{-}\right\rangle$(see Supplementary Information S3 for the evolution of the energy spectrum). To demonstrate this deterministic preparation, we take as an example $\mathcal{E}_{\mathrm{p}}(t)=\mathcal{E}_{\mathrm{p}}^{0}\left[1-\exp \left(-t^{4} / \tau^{4}\right)\right]$ such that for $t \gg \tau, \mathcal{E}_{\mathrm{p}}(t) \sim \mathcal{E}_{\mathrm{p}}^{0}=4 K$ with $\tau K=5$ to satisfy the adiabatic condition. Without photon loss, the fidelity of the resulting cat state at $t=6.5 / K$ is $99.9 \%$ while for $K / K=250^{31}$ the fidelity at $t=$ $6.5 / K$ is reduced to $98.3 \%$.

\section{High-fidelity nonadiabatic initialization}

To speed up the adiabatic preparation described above, we follow the approach of transitionless driving. ${ }^{21,32,33}$ This technique relies on introducing an auxiliary counter-adiabatic Hamiltonian, $\hat{H}^{\prime}(t)=i\left[\left|\dot{\psi}_{n}(t)\right\rangle\left\langle\psi_{n}(t)|-| \psi_{n}(t)\right\rangle\left\langle\dot{\psi}_{n}(t)\right|\right]$, chosen such that the system follows the instantaneous eigenstate $\left|\psi_{n}(t)\right\rangle$ of the system Hamiltonian $\hat{H}_{0}(t)$ even under nonadiabatic changes of the system parameters. This idea has been experimentally demonstrated with Bose-Einstein condensates in optical lattices ${ }^{34}$ and nitrogen vacancy centers in diamonds. ${ }^{35}$ Here, to prepare the even parity cat-state $\left|\mathcal{C}_{a_{0}(t)}^{+}\right\rangle$, the required counter-adiabatic Hamiltonian is

$$
\hat{H}^{\prime}(t)=i \frac{\dot{a}_{0}(t)}{\mathcal{N}_{a_{0}(t)}^{-}}\left[\hat{a}^{\dagger}\left|\mathcal{C}_{a_{0}(t)}^{-}\right\rangle\left\langle\mathcal{C}_{a_{0}(t)}^{+}|-| \mathcal{C}_{a_{0}(t)}^{+}\right\rangle\left\langle\mathcal{C}_{a_{0}(t)}^{-}\right| \hat{a}\right] .
$$


While exact, this does not correspond to an easily realizable Hamiltonian. It can, however, be approximated to (see Methods),

$$
\hat{H}^{\prime}(t) \sim i \frac{\dot{a}_{0}(t)}{\mathcal{N}_{a_{0}(t)}^{-}\left[1+2 a_{0}(t)\right]}\left(\hat{a}^{\dagger 2}-\hat{a}^{2}\right),
$$

which can be implemented with an additional two-photon drive orthogonal to $\mathcal{E}_{p}(t)$. As an illustration of this method, we reconsider the example presented in the previous section now with the much shorter evolution time of $\tau=1 / K$. As shown by the Wigner function in Fig. 2a, without the additional two-photon drive of Eq. (5), the state at time $t=1.37 / \mathrm{K}$ is highly distorted. On the other hand, and as illustrated in Fig. 2b, initialization with the appropriate auxiliary orthogonal two-photon drive leads to cat-state fidelities of $99.9 \%$ with $K=0$ and $99.5 \%$ with $K=K / 250$. In other words, we find that the protocol is made $\sim 5$ times faster by the addition of the orthogonal drive, thereby improving the fidelity in the presence of single-photon loss. These results, obtained with the analytical expression of Eq. (5), can be further improved upon using numerical optimal control. ${ }^{36}$ For example, using the approach recently described in ref. 37 , we find that cat states can be initialized in times as short as $0.3 / K$ with fidelity 99.995\% (see Supplementary Information S4). Adiabatic cat state preparation with two-photon driving was also investigated in a noiseless idealized KNR. ${ }^{38,} 39$ These previous studies lack the crucial examination of eigenspace distortion that arise, as will be discussed below, during gate operations and fall short of accounting for higher-order nonlinearities that exist in realistic physical implementations.

\section{Realization with superconducting circuits}

One standard approach to realize a two-photon driven Kerrnonlinear resonator is to terminate a $\lambda / 4$ microwave resonator with a flux-pumped SQUID, a device known as a josephson parametric amplifier (JPA) ${ }^{40-42}$ (see also Supplementary Information S5). The non-linear inductance of the SQUID induces a Kerr nonlinearity and a two-photon drive is introduced by the modulation of the flux-pump at twice the resonator frequency. As an illustrative example, with a realistic JPA Kerr-nonlinearity of $K / 2 \pi=750 \mathrm{KHz}$ it is possible to encode a cat state with $a_{0}=2$ in a time $0.3 / K=63.6 \mathrm{~ns}$ using the transitionless driving approach with numerically optimized pulse shape. We have, moreover, simulated the cat state initialization protocol under the exact Hamiltonian of a JPA including the full Josephson junction cosine potential.
As discussed in the Supplementary Information S5, the results are essentially unchanged showing that the strong state confinement to the coherent states $\left| \pm a_{0}\right\rangle$ is also robust against higher-order nonlinearities that will arise in a circuit implementation of these ideas. An alternative realization of the two-photon driven KNR is based on a 3D microwave cavity coupled to a Josephson junction. The non-linear inductance of the junction induces a Kerr nonlinearity, while a microwave drive on the junction at the 3D cavity frequency introduces the required two-photon drive. ${ }^{14,} 20$

We note that the engineered dissipation approach of Refs 14, 20 also relies on a two-photon drive to achieve confinement to the subspace of two coherent states with opposite phases. There, the two-photon drives is used to induce two-photon loss at a rate $\kappa_{2 \mathrm{ph}}$. This rate must be made large with respect to the single-photon loss rate $\kappa$ for high fidelity initialization of cat states, something which is challenging experimentally. In contrast, the present approach does not rely on dissipation but rather takes advantage of the large Kerr-nonlinearity $K$ that is easily realized in superconducting quantum circuits. Even in the presence of two-photon loss, robust confinement is obtained if $K>K_{2 \mathrm{ph}}$, a condition that is easily satisfied in practice.

Stabilization of cat states against Kerr induced rotation and dephasing

Even with high-fidelity cat state preparation, it is important to limit the unwanted phase evolution and dephasing arising from Kerr nonlinearity and single-photon loss. We now illustrate, with two examples, how a two-photon drive of appropriate amplitude and phase can correct this unwanted evolution. First consider a resonator deterministically initialized to $\left|\mathcal{C}_{a}^{+}\right\rangle$. Fig. 3a-c illustrates the evolution of this initial state in the absence of two-photon drive. Kerr nonlinearity leads to deterministic deformation of the state $^{6,8}$ which, in the presence of single-photon loss, also induces additional dephasing. This results in a reduction of the contrast of the Wigner function fringes, a reduction of the separation of the cat components and a broadening of these components. As a result, the fidelity of $\left|\mathcal{C}_{a}^{ \pm}\right\rangle$decreases faster in a KNR than in a linear resonator (see Supplementary Information S9). While the deterministic phase rotation can be accounted for and corrected in a simple way, this is not the case for Kerr-induced dephasing. ${ }^{24}$ Fig. $3 \mathrm{~d}$-f illustrates the same initial cat state now stabilized against Kerr-induced rotation and dephasing by the application of a two-photon drive. This drive is chosen such that its amplitude
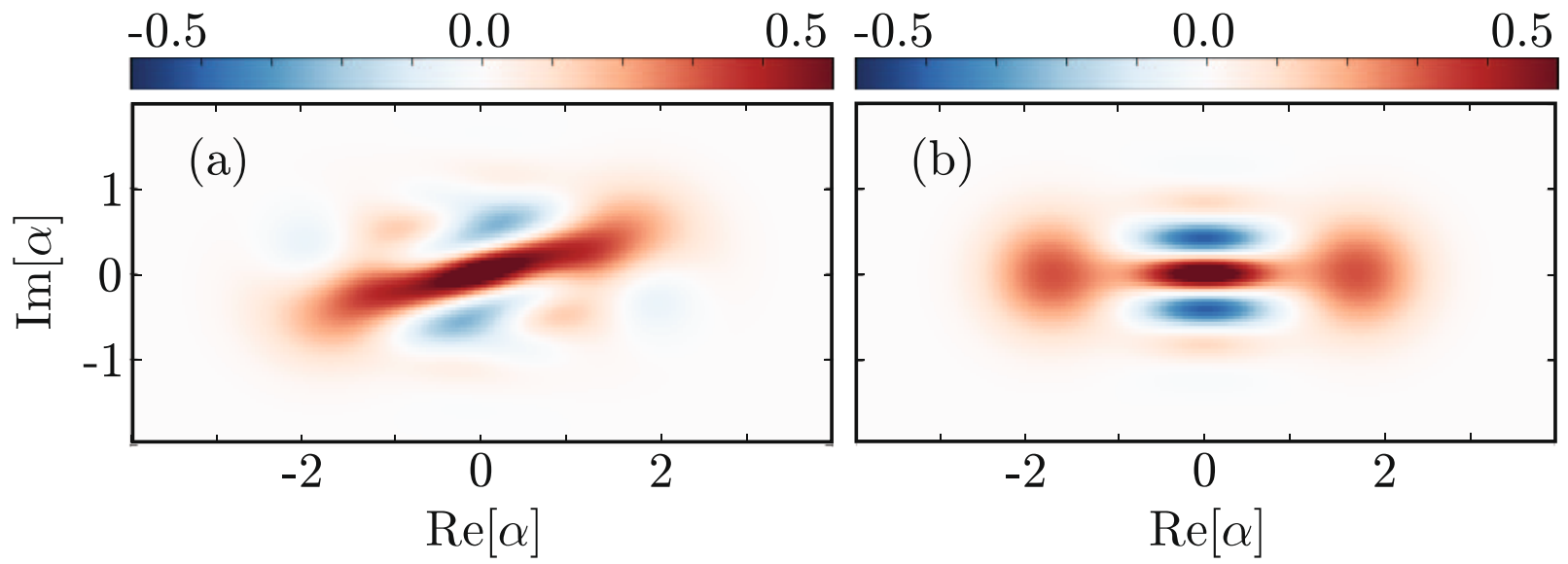

Fig. 2 Wigner function for a KNR initialized in vacuum $|0\rangle$ and driven by a a single parametric drive $\mathcal{E}_{\mathrm{p}}=\mathcal{E}_{\mathrm{p}}^{0}\left[1-\exp \left(-t^{4} / \tau^{4}\right)\right]$ b with two orthogonal parametric drives, $\mathcal{E}_{\mathrm{p}}=\mathcal{E}_{\mathrm{p}}^{0}\left[1-\exp \left(-t^{4} / \tau^{4}\right)\right]$ and $\mathcal{E}_{\mathrm{p}}^{\prime}(t)=i \dot{a}_{0}(t) N_{a_{0}(t)}^{-} /\left(1+2 a_{0}(t)\right)$, where $a_{0}(t)=\sqrt{\mathcal{E}_{\mathrm{p}}(t) / K}$. The Wigner function is plotted at time $t=1.37 \tau$, with $\tau=1 / K, \mathcal{E}_{\mathrm{p}}^{0}=4 K$. Without the auxiliary drive $\mathcal{E}_{\mathrm{p}}^{\prime}$ the non-adiabatic driving of the system results in an imperfect cat state. However, the auxiliary drive induces counter-adiabatic terms, resulting in near perfect initialization of the cat state. At $t=1.3 \tau$, the fidelity with respect to $\left|\mathcal{C}_{2}^{+}\right\rangle$is $99.9 \%$ for $K=0$ and $99.5 \%$ for $K / K=250$ 


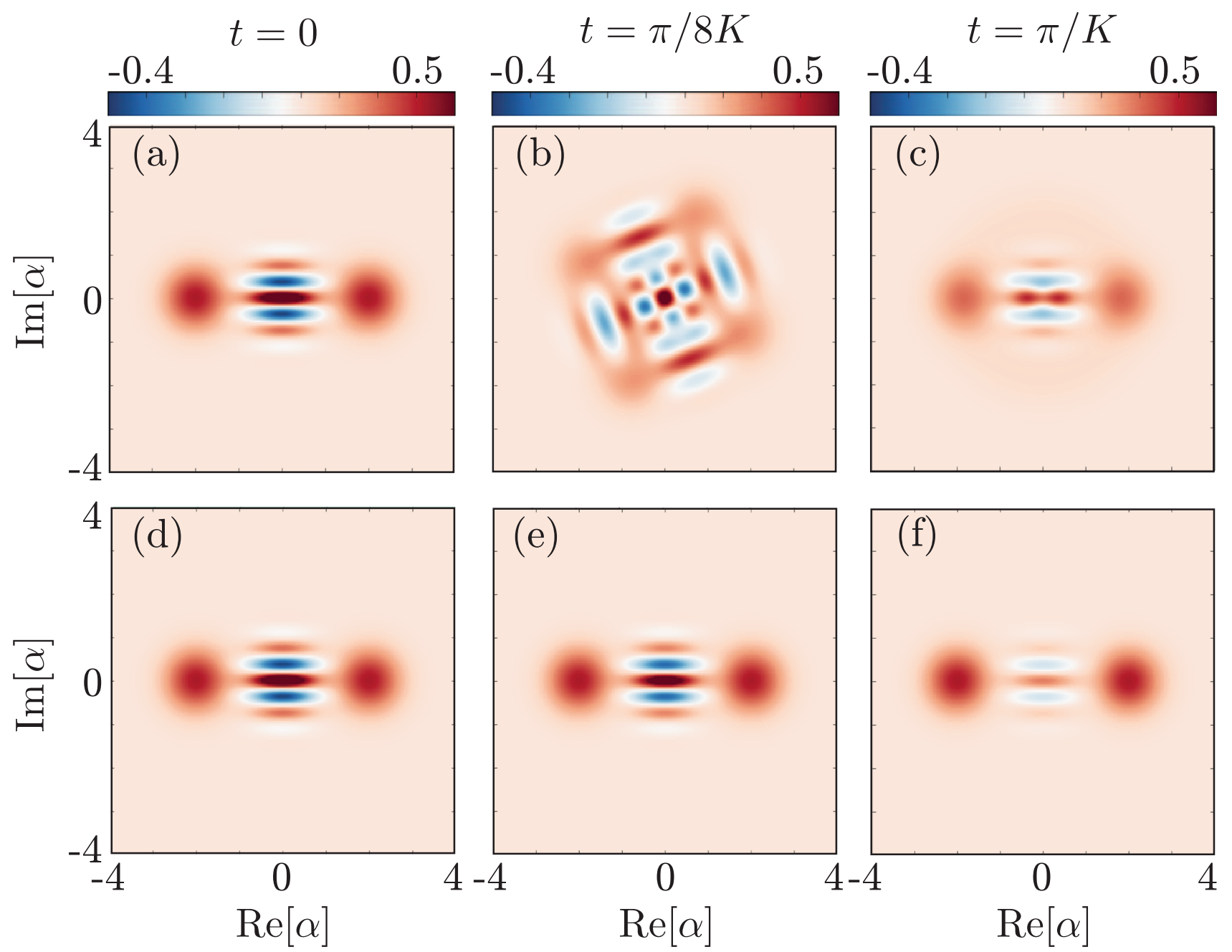

Fig. 3 Wigner functions at different times for a lossy KNR initialized to $\left|\mathcal{C}_{2}^{+}\right\rangle$without a-c and with d-f two-photon driving. $K / K=20$ and $\mathcal{E}_{p} \sim 4 K$

$\mathcal{E}_{p}$ satisfies Eq. (3). The confinement in phase space provided by the two-photon driven KNR prevents amplitude damping of the stabilized coherent states $\left| \pm a_{0}\right\rangle$. As a result, the cat state fidelity in this system decreases more slowly in time that in a linear resonator. As a simple extension, we also find that it is possible to stabilize coherent states against Kerr-induced rotation and dephasing (see Supplementary Information S1). These somewhat counterintuitive results shows that, even in the presence of loss, a Gaussian drive (i.e., two-photon drive) can completely remove the highly non-Gaussian effect of a Kerr nonlinearity.

As a second example, we consider the qcMAP gate for cat state preparation, a protocol that relies on the strong dispersive qubitresonator interaction that is realized in circuit QED..$^{13}$ In practice, this strong interaction is accompanied by a qubit-induced Kerr nonlinearity of the field. ${ }^{22,}{ }^{23}$ As a result, even at modest $a$, cat states suffer from deformations. ${ }^{15}$ This effect is illustrated in Fig. $4 a$, $b$ which shows the cat state obtained from qcMAP under ideal dispersive interaction (ignoring any Kerr nonlinearities) and under the full Jaynes-Cummings Hamiltonian, respectively. Distortions are apparent in panel b) and the fidelity to the ideal cat is reduced to $94.1 \%$. In contrast, Fig. 4c shows the same Wigner function prepared using the qCMAP protocol with the full Jaynes-Cummings interaction and an additional two-photon drive. The resulting fidelity is $99.4 \%$, approaching the fidelity of 99.8\% obtained under the ideal, but not realistic, dispersive Hamiltonian. The amplitude of the two-photon drive was optimized numerically to take into account the qubit-induced Kerr nonlinearity (see Supplementary Information S10).

Universal quantum logic gates

Following the general approach of Ref. 14, we now turn to the realization of a universal set of gates in the two-photon driven KNR. Taking advantage of the quasi-orthogonality of coherent states for large $a$, both the $\left\{\left|\mathcal{C}_{\alpha_{0}}^{ \pm}\right\rangle\right\}$and the $\left\{\left| \pm a_{0}\right\rangle\right\}$ basis can be used as logical states. Here, we choose the latter which we will now refer to as $\{|\overline{0}\rangle,|\overline{1}\rangle\}$. With this choice, a logical Z rotation can be realized by lifting the degeneracy between $|\overline{0}\rangle$ and $|\overline{1}\rangle$ using a single-photon drive in combination to $\hat{H}_{0}: \hat{H}_{z}=\hat{H}_{0}+\mathcal{E}_{\mathrm{z}}\left(\hat{a}^{\dagger}+\hat{a}\right)$. For $\left|\mathcal{E}_{\mathrm{z}}\right| \ll\left|4 K a_{0}^{3}\right|$ and $\mathcal{E}_{\mathrm{p}}$ real, the only effect of this additional drive is to lift the degeneracy by $\delta_{z}=4 \mathcal{E}_{z} a_{0}$ (Supplementary Information S6). Indeed, in the space spanned by $\{|\overline{0}\rangle,|\overline{1}\rangle\}$, the single-photon drive Hamiltonian can be expressed as $\bar{I} \mathcal{E}_{\mathrm{z}}\left(\hat{a}^{\dagger}+\hat{a}\right)$ $\bar{l}=\delta_{z} \bar{\sigma}_{z} / 2$, where $\bar{l}=|\overline{0}\rangle\langle\overline{0}|+| \overline{1}\rangle\langle\overline{1}|$ and $\bar{\sigma}_{z}=|\overline{0}\rangle\langle\overline{0}|-| \overline{1}\rangle\langle\overline{1}|$. Numerical simulations of this process for a time $\tau=1 / \delta_{z^{\prime}}$ corresponding to the gate $\hat{R}_{\bar{z}}(\pi)$, with the resonator initialized to $\left|\mathcal{C}_{a_{0}}^{+}\right\rangle$and the choices $\mathcal{E}_{\mathrm{p}}=4 K, \mathcal{E}_{\mathrm{z}}=0.8 \mathrm{~K}$ leads to a fidelity of $99.9 \%$ with $K=0$ and $99.5 \%$ for $K / K=250$. Increasing $\mathcal{E}_{\text {z' }}$ so that the condition $\left|\mathcal{E}_{z}\right| \ll\left|4 K a_{0}^{3}\right|$ is no longer satisfied, distorts the eigenstates and as a consequence the fidelity of the gate decreases. The dependence of the gate fidelity on the strength of the single photon drive is examined further in Supplementary Information S8. A similar scheme for single-qubit rotation has 


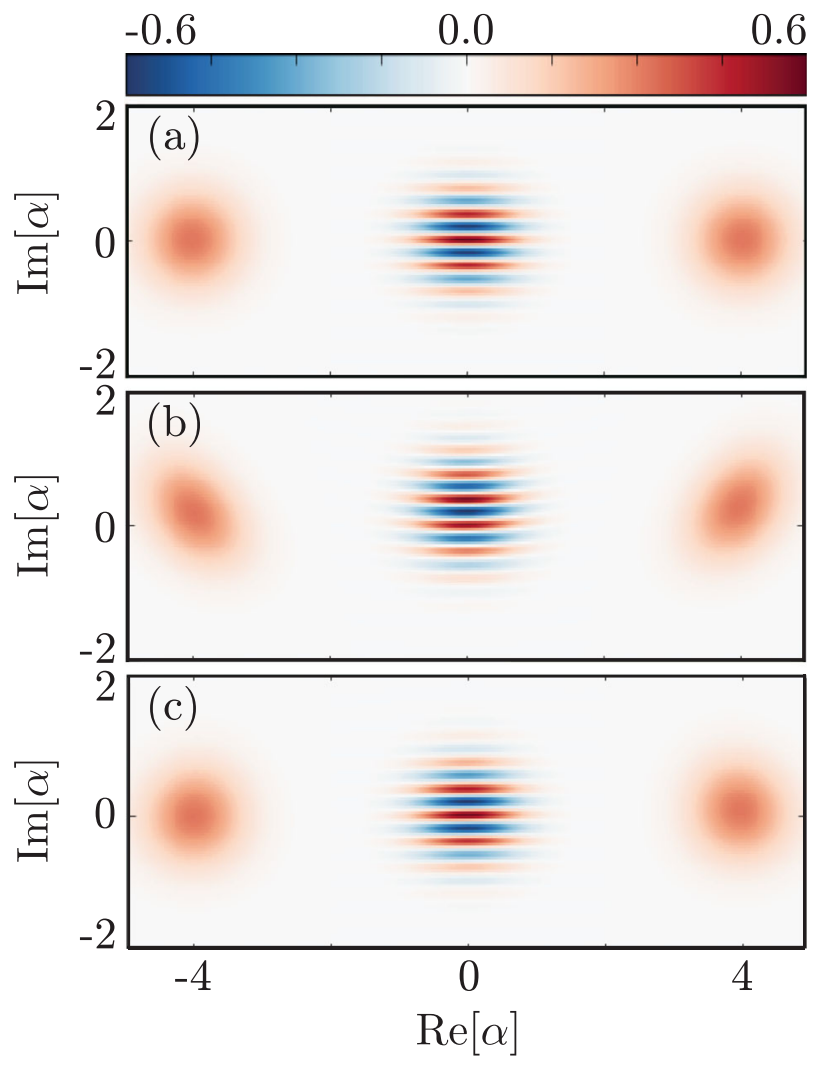

Fig. 4 Wigner function of final state under qcMAP gate with a ideal dispersive Hamiltonian, $\mathbf{b}$ full Jaynes-Cummings Hamiltonian and $\mathbf{c}$ full Jaynes-Cummings Hamiltonian and two-photon drive

been proposed for resonators with engineered two-photon loss. ${ }^{14}$ However, this requires the drive strength to be significantly smaller than the two-photon loss rate which is typically of the order of $50-100 \mathrm{kHz},{ }^{20}$ thereby leading to long-gate times.

The strong state confinement resulting from the two-photon driven KNR prevents population transfer between the two logical states, making it difficult to implement $X$ rotations. One approach to implement $\hat{R}_{\bar{x}}(\pi / 2)$ is to temporarily remove the two-photon drive and let the state evolve under the Kerr Hamiltonian. ${ }^{14}$ Alternatively, an arbitrary $\hat{R}_{\bar{x}}(\theta)$ can be realized by introducing a detuning between the two-photon drive and the resonator corresponding to the Hamiltonian $\hat{H}_{\mathrm{x}}=\hat{H}_{0}+\delta_{x} \hat{a}^{\dagger} \hat{a}$. For $\delta_{x} \ll$ $2 \mathcal{E}_{\mathrm{p}}$ (Supplementary Information S7), this can be understood by projecting the number operator in the logical basis: $\bar{l} \hat{a}^{\dagger} \hat{a} \bar{l}=$ $\left|a_{0}\right|^{2} \bar{l}-\left|a_{0}\right|^{2} e^{-2\left|a_{0}\right|^{2}} \bar{\sigma}_{x}$. Despite the exponential reduction with $a_{0}$ of the effective Rabi frequency, high-fidelity rotations can be achieved. Numerical simulations on a resonator initialized to $|\overline{0}\rangle$ and for a time $\tau=\pi /\left(4 \delta_{X}\left|a_{0}\right|^{2} e^{-2\left|\alpha_{0}\right|^{2}}\right)$, corresponding to the gate $\hat{R}_{\bar{x}}(\pi / 2)$, leads to a fidelity of $99.7 \%$ for $K=0$ and $98.6 \%$ for $K / K=$ 250 with $\mathcal{E}_{p}=K$ and $\delta_{x}=K / 3$. Similarly to the $Z$ rotations, the fidelity of the $X$ gate also decreases if the condition $\delta_{x} \ll 2 \mathcal{E}_{\mathrm{p}}$ is not met (see Supplementary Information S8).

To complete the set of universal gates, an entangling gate between the field stored in two distinct resonators, or alternatively two modes of a single resonator, is needed. From the discussion on the $\hat{R}_{\bar{z}}(\theta)$ gate, it follows that a $\bar{\sigma}_{\mathrm{z} 1} \bar{\sigma}_{\mathrm{z} 2}$ interaction between the two fields is obtained by linearly coupling the two-photon driven KNRs, the Hamiltonian now reading $\hat{H}_{\mathrm{zz}}=\hat{H}_{01}+\hat{H}_{02}+$ $\mathcal{E}_{\text {zz }}\left(\hat{a}_{1}^{\dagger} \hat{a}_{2}+\hat{a}_{1} \hat{a}_{2}^{\dagger}\right)$. To simplify the discussion, the two resonators are assumed to be identical with $\hat{H}_{0 i}=-K \hat{a}_{i}^{\dagger 2} \hat{a}_{i}^{2}+\mathcal{E}_{\mathrm{p}}\left(\hat{a}_{i}^{\dagger 2}+\hat{a}_{i}^{2}\right)$. Expressed in the logical basis, the bilinear coupling Hamiltonian takes the desired form $\delta_{z z} \bar{\sigma}_{z 1} \bar{\sigma}_{z 2}$, with $\delta_{z z}=4 \mathcal{E}_{z z}\left|a_{0}\right|^{2}$. In order to demonstrate this gate, we simulate the master equation under $\hat{H}_{z z}$ with the resonators initialized to the product state $\left|\mathcal{C}_{a_{0}}^{+}\right\rangle \otimes\left|\mathcal{C}_{a_{0}}^{+}\right\rangle$ and $\mathcal{E}_{p}=4 K, \mathcal{E}_{\mathrm{zz}}=K / 5$. As expected, the initial product state is transformed to the maximally entangled state $(|\overline{0}, \overline{0}\rangle+i|\overline{0}, \overline{1}\rangle+$ $i|\overline{1}, \overline{0}\rangle+|\overline{1}, \overline{1}\rangle) / 2$ at $t=\pi / 2 \delta_{z z}$ with fidelity $F=99.99 \%$ for $k=0$ and $F=94 \%$ for $K / K=250$. Supplementary Information S8 examines the fidelity dependence on the strength of the two-photon drive. Similar approaches for $Z$ rotations and entangling gate have been presented before, ${ }^{39}$ however without the crucial analysis of the restrictions on the amplitude of the single-photon drive and strength of the single-photon exchange coupling.

\section{DISCUSSION}

To summarize, we have shown that, in the presence of a two-photon drive, the eigenspace of a KNR can be engineered to be two out-ofphase coherent states that are robust against single-photon loss. This quantum state engineering offers a practical way to correct the undesirable effects of Kerr nonlinearity in applications such as the qcMAP gate. We have also described protocols for fast-high fidelity initialization and manipulation cat states for quantum information processing. This approach offers significant improvements over previous techniques based on dispersive qubit-resonator interactions or reservoir engineering. These results suggest a minimal approach to prepare and manipulate cat states of the field of a microwave resonator using only a Josephson parametric amplifier and are of immediate practical importance for realization of a scalable, hardware efficient platform for quantum computation. Furthermore, the observation that $n$ coherent states are the degenerate eigenstates of the Hamiltonian $\hat{H}=-K \hat{a}^{\dagger n} \hat{a}^{n}+\mathcal{E}_{\mathrm{p}}\left(\hat{a}^{\dagger n}+\hat{a}^{n}\right)$ provides an approach for initializing $n$-component cat states. Such a Hamiltonian could be implemented with a JPA, in which the cosine potential of a Josephson junction supplies the required nonlinearity and flux modulation through the SQUID loop at $n$-times the resonator frequency triggers the $n$-photon drive. Our work opens new directions for the JPA as a powerful device for implementing quantum algorithms based on multi-component cats.

\section{METHODS}

Eigenstates of the $n$-photon driven Hamiltonian

Consider the Hamiltonian

$\hat{H}_{n}=-K \hat{a}^{\dagger n} \hat{a}^{n}+\left(\mathcal{E}_{\mathrm{p}} \hat{a}^{\dagger n}+\mathcal{E}_{\mathrm{p}}^{*} \hat{a}^{n}\right)=-K\left(\hat{a}^{\dagger n}-\frac{\mathcal{E}_{\mathrm{p}}^{*}}{K}\right)\left(\hat{a}^{n}-\frac{\mathcal{E}_{\mathrm{p}}}{K}\right)+\frac{\left|\mathcal{E}_{\mathrm{p}}\right|^{2}}{K}$.

The second form makes it clear that the coherent state $|a\rangle$ with $a^{n}-\mathcal{E}_{p} / K=0$ is an eigenstate of $\hat{H}_{n}$. Thus, in general, there are $n$ coherent states that are the degenerate eigenstates of $\hat{H}_{n}$ with energy $\left|\mathcal{E}_{p}\right|^{2} / K$.

Effective Hamiltonian and steady-state

Under single-photon loss, the system's master equation takes the form ${ }^{26}$

$\dot{\hat{\rho}}=-i\left(\hat{H}_{\text {eff }} \hat{\rho}-\hat{\rho} \hat{H}_{\text {eff }}^{\dagger}\right)+\kappa \hat{a} \hat{\rho} \hat{a}^{\dagger}$,

where $\hat{H}_{\text {eff }}=\hat{H}_{0}-i \kappa \hat{a}^{\dagger} \hat{a} / 2$ and $\hat{H}_{0}=-K \hat{a}^{\dagger} \hat{a}^{\dagger} \hat{a} \hat{a}+\left(\mathcal{E}_{\mathrm{p}} \hat{a}^{\dagger 2}+\mathcal{E}_{\mathrm{p}}^{*} \hat{a}^{2}\right)$. Under displacement transformation $D\left(a_{0}\right)=\exp \left(a_{0} \hat{a}^{\dagger}-a_{0} \hat{a}\right), \hat{H}_{\text {eff }}$ reads

$$
\begin{aligned}
\hat{H}_{\text {eff }}^{\prime}= & D^{\dagger}\left(a_{0}\right) \hat{H}_{\text {eff }} D\left(a_{0}\right) \\
& =\left[\left(-2 K a_{0}^{2} a_{0}^{*}+2 \mathcal{E}_{\mathrm{p}} a_{0}^{*}-i \frac{k}{2} a_{0}\right) \hat{a}^{\dagger}+\text { h.c. }\right] \\
& +\left[\left(-K a_{0}^{2}+\mathcal{E}_{\mathrm{p}}\right) \hat{a}^{\dagger 2}+\text { h.c. }\right]-4 K|a|^{2} \hat{a}^{\dagger} \hat{a}-i \frac{k}{2} \hat{a}^{\dagger} \hat{a} \\
& -K \hat{a}^{\dagger 2} \hat{a}^{2}-\left(2 K a_{0} \hat{a}^{\dagger 2} a+\text { h.c. }\right),
\end{aligned}
$$

where we have dropped the constant term $E=-K\left|a_{0}\right|^{4}+\mathcal{E}_{\mathrm{p}}^{*} a_{0}^{2}+$ $\mathcal{E}_{\mathrm{p}} a_{0}^{* 2}-i \kappa\left|a_{0}\right|^{2} / 2$ that represents a shift in energy of the non-Hermitan effective Hamiltonian. We take $a_{0}$ to satisfy

$-2 K a_{0}^{2} a_{0}^{*}+2 \mathcal{E}_{\mathrm{p}} a_{0}^{*}-i \frac{K}{2} a_{0}=0$, 
such as to cancel the first line of $\hat{H}_{\text {eff }}^{\prime}$ which now reads

$$
\begin{aligned}
\hat{H}_{\text {eff }}^{\prime}= & {\left[\left(-K a_{0}^{2}+\mathcal{E}_{\mathrm{p}}\right) \hat{a}^{\dagger 2}+\text { h.c. }\right] } \\
& -\left(4 K\left|a_{0}\right|^{2}+i \frac{K}{2}\right) \hat{a}^{\dagger} \hat{a}-K \hat{a}^{\dagger 2} \hat{a}^{2}-2 K a_{0} \hat{a}^{\dagger 2} \hat{a}-2 K a_{0}^{*} \hat{a}^{\dagger} \hat{a}^{2} .
\end{aligned}
$$

Eq. (9) is satisfied for $a_{0}=0, \pm r_{0} e^{i \theta_{0}}$ where

$r_{0}=\left(\frac{4 \mathcal{E}_{\mathrm{p}}^{2}-\kappa^{2} / 4}{4 K^{2}}\right)^{1 / 4}, \quad \theta_{0}=\frac{1}{2} \tan ^{-1}\left(\frac{\kappa}{\sqrt{16 \mathcal{E}_{\mathrm{p}}^{2}-\kappa^{2}}}\right)$.

For $a_{0}=0$, the first two terms of Eq. (10) represent a near resonant parametric drive of strength $\mathcal{E}_{\mathrm{p}}$. This results in large fluctuations making the system unstable around $a_{0}=0$. On the other hand, for $a_{0}= \pm r_{0} e^{i \theta_{0}}$, the displaced effective Hamiltonian can be rewritten as

$$
\begin{aligned}
\hat{H}_{\text {eff }}^{\prime}= & \frac{1}{2}\left[i \frac{K a_{0}}{2 a_{0}^{*}} \hat{a}^{\dagger 2}+\text { c.c. }\right]-\left(4 K\left|a_{0}\right|^{2}+i \frac{K}{2}\right) \hat{a}^{\dagger} \hat{a} \\
& -K \hat{a}^{\dagger 2} \hat{a}^{2}-2 K a_{0} \hat{a}^{\dagger 2} \hat{a}-2 K a_{0}^{*} \hat{a}^{\dagger} \hat{a}^{2} .
\end{aligned}
$$

The first two terms of Eq. (12) now represent a parametric drive whose amplitude has an absolute value of $k / 2$ and is detuned by $4 K\left|a_{0}\right|^{2}+i K / 2 \approx 4 K\left|a_{0}\right|^{2}$. In other words, the effect of single-photon loss $K$ is to squeeze the field around $a_{0}= \pm r_{0} e^{i \theta_{0}}$ leading to increased quantum fluctuations. For $K \ll 8 K\left|a_{0}\right|^{2}$, the resulting fluctuations are, however, small and $|0\rangle$ remains an eigenstate in the displaced frame. This implies that, back in the lab frame, $\left| \pm a_{0}\right\rangle$ are the degenerate eigenstates of $\hat{H}_{\text {eff. }}$. As a result, $\hat{\rho}_{\mathrm{s}}=\left(\left|a_{0}\right\rangle\left\langle a_{0}|+|-a_{0}\right\rangle\left\langle-a_{0}\right|\right) / 2$ is a steady-state of Eq. (7). It is, moreover, the unique steady-state of this system since only the two eigenstates $\left| \pm a_{0}\right\rangle$ of the effective Hamiltonian are also invariant under the quantum jump operator $\hat{a} .{ }^{43}$ Following the analysis here, it is also possible to characterize the effect of, for example, single-photon drive, detuning, etc (see Supplementary Notes).

\section{Cat state decoherence under single-photon loss}

In the previous section, we saw that the coherent states $\left| \pm a_{0}\right\rangle$ are eigenstates of the two-photon driven KNR even in the presence of singlephoton loss. However, this loss channel results in decoherence of superpositions of these two states, i.e., of cat states. Indeed, the last term of the master equation Eq. (7), $k \hat{a} \hat{\rho} \hat{a}^{\dagger}$, transforms the even parity cat state $\left|\mathcal{C}_{\alpha_{0}}^{+}\right\rangle$to the odd parity cat state $\left|\mathcal{C}_{\alpha_{0}}^{-}\right\rangle$and vice-versa. This results in decoherence and reduction in the contrast of the Wigner function fringes. The rate of this phase decay is given by $\gamma=\kappa\left|a_{0}-\left(-a_{0}\right)\right|^{2} / 2=2 \kappa\left|a_{0}\right|^{2}$.

Consider for example the cat state initialization protocol with $\mathcal{E}_{\mathrm{p}}=\mathcal{E}_{\mathrm{p}}^{0}$ $\left[1-\exp \left(-t^{4} / \tau^{4}\right)\right]$ and $\mathcal{E}_{\mathrm{p}}^{0}=4 K$ so that $a_{0}(t)=2 \sqrt{\left[1-\exp \left(-t^{4} / \tau^{4}\right)\right]}$. The phase error during this initialization can be estimated to be $\exp \left(-2 \int \kappa\left|a_{0}(t)\right|^{2} \mathrm{~d} t\right)=0.016$, resulting in a fidelity of $98.4 \%$. This estimate compares very well with the numerically estimated fidelity quoted earlier in the manuscript (98.3\%).

Additional Hamiltonian for faster than adiabatic initialization of cat state

Consider the exact Hamiltonian in Eq. (4) required for transitionless quantum driving. At short times $t \sim 0$, we have that $a_{0}(t) \sim 0$ and as a result $\left|\mathcal{C}_{0}^{+}\right\rangle \sim|n=0\rangle$ and $\left|\mathcal{C}_{0}^{-}\right\rangle \sim|n=1\rangle$. Therefore, $\left[\hat{a}^{\dagger}\left|\mathcal{C}_{\alpha_{0}(t)}^{-}\right\rangle\left\langle\mathcal{C}_{\alpha_{0}(t)}^{+}|-| \mathcal{C}_{a_{0}(t)}^{+}\right\rangle\right.$ $\left.\left\langle\mathcal{C}_{a_{0}(t)}^{-}\right| \hat{a}\right] \sim\left[\hat{a}^{\dagger}|1\rangle\langle 0|-| 0\rangle\langle 1| \hat{a}\right] \sim \hat{a}^{\dagger 2}-\hat{a}^{2}$. On the contrary, at long time the coherent states become quasi-orthogonal and a single photon jump leads to the transition between even and odd photon number cat states. This suggests that if $a_{0}(t) \gg 1$, it is possible to approximate $\left[\hat{a}^{\dagger}\left|\mathcal{C}_{\alpha_{0}(t)}^{-}\right\rangle\left\langle\mathcal{C}_{\alpha_{0}(t)}^{+}|-| \mathcal{C}_{a_{0}(t)}^{+}\right\rangle\left\langle\mathcal{C}_{\alpha_{0}(t)}^{-}\right| \hat{a}\right] \sim\left(\hat{a}^{\dagger 2}-\hat{a}^{2}\right) / 2 a_{0}(t)$ in the restricted coherent state basis. Therefore, in order to reconcile both short and longtime behavior, we choose, $\left[\hat{a}^{\dagger}\left|\mathcal{C}_{\alpha_{0}(t)}^{-}\right\rangle\left\langle\mathcal{C}_{\alpha_{0}(t)}^{+}|-| \mathcal{C}_{\alpha_{0}(t)}^{+}\right\rangle\left\langle\mathcal{C}_{\alpha_{0}(t)}^{-}\right| \hat{a}\right] \sim\left(\hat{a}^{\dagger 2}-\right.$ $\left.\hat{a}^{2}\right) /\left[1+2 a_{0}(t)\right]$ to obtain Eq. (5).

\section{ACKNOWLEDGEMENTS}

We thank M. Mirrahimi, A. Grimsmo, and C. Andersen for useful discussions. This work was supported by the Army Research Office under Grant W911NF-14-1-0078 and by
NSERC. This research was undertaken thanks in part to funding from the Canada First Research Excellence Fund.

\section{AUTHOR CONTRIBUTIONS}

S.P. and A.B. conceived and developed the idea, S.B. contributed to the circuit analysis and developed the code based on GRAPE for pulse optimization.

\section{COMPETING INTERESTS}

The authors declare that they have no competing interests.

\section{REFERENCES}

1. Dykman, M. Fluctuating nonlinear oscillators: from nanomechanics to quantum superconducting circuits (OUP Oxford, 2012).

2. Siddiqi, l. et al. Direct observation of dynamical bifurcation between two driven oscillation states of a josephson junction. Phys. Rev. Lett. 94, 027005 (2005).

3. Yurke, B. et al. Observation of 4.2-k equilibrium-noise squeezing via a josephsonparametric amplifier. Phys. Rev. Lett. 60, 764 (1988).

4. Castellanos-Beltran, M. A., Irwin, K. D., Hilton, G. C., Vale, L. R. \& Lehnert, K. W. Amplification and squeezing of quantum noise with a tunable josephson metamaterial. Nat. Phys. 4, 929-931 (2008).

5. Munro, W. J., Nemoto, K. \& Spiller, T. P. Weak nonlinearities: a new route to optical quantum computation. New J. Phys. 7, 137 (2005). http://stacks.iop.org/1367$2630 / 7 / \mathrm{i}=1 / \mathrm{a}=137$.

6. Yurke, B. \& Stoler, D. Generating quantum mechanical superpositions of macroscopically distinguishable states via amplitude dispersion. Phys. Rev. Lett. 57, 13-16 (1986).

7. Boyd, R. W. Nonlinear optics (Academic press, 2003).

8. Kirchmair, G. et al. Observation of quantum state collapse and revival due to the single-photon kerr effect. Nature 495, 205-209 (2013).

9. Zurek, W. H. Decoherence, einselection, and the quantum origins of the classical. Rev. Modern Phys. 75, 715 (2003).

10. Munro, W. J., Nemoto, K., Milburn, G. J. \& Braunstein, S. L. Weak-force detection with superposed coherent states. Phys. Rev. A 66, 023819 (2002).

11. Ralph, T., Gilchrist, A., Milburn, G. J., Munro, W. J. \& Glancy, S. Quantum computation with optical coherent states. Phys. Rev. A 68, 042319 (2003).

12. Albert, V. V. et al. Holonomic quantum control with continuous variable systems. Phys. Rev. Lett. 116, 140502 (2016).

13. Leghtas, Z. et al. Deterministic protocol for mapping a qubit to coherent state superpositions in a cavity. Phys. Rev. A 87, 042315 (2013).

14. Mirrahimi, M. et al. Dynamically protected cat-qubits: a new paradigm for universal quantum computation. New J. Phys. 16, 045014 (2014).

15. Vlastakis, B. et al. Deterministically encoding quantum information using 100photon schrödinger cat states. Science 342, 607-610 (2013).

16. Leghtas, Z. et al. Hardware-efficient autonomous quantum memory protection. Phys. Rev. Lett. 111, 120501 (2013).

17. Wang, C. et al. A schrodinger cat living in two boxes. Preprint at arXiv:1601.05505 (2016).

18. Ofek, N. et al. Demonstrating quantum error correction that extends the lifetime of quantum information. Preprint at arXiv:1602.04768 (2016).

19. Gambetta, J. et al. Qubit-photon interactions in a cavity: measurement-induced dephasing and number splitting. Phys. Rev. A (Atomic, Molecular, and Optical Physics) 74, 042318 (2006). http://link.aps.org/abstract/PRA/v74/e042318.

20. Leghtas, Z. et al. Confining the state of light to a quantum manifold by engineered two-photon loss. Science 347, 853-857 (2015).

21. Berry, M. Transitionless quantum driving. J. Phys. A: Mathematical and Theoretical 42, 365303 (2009)

22. Boissonneault, M., Gambetta, J. M. \& Blais, A. Dispersive regime of circuit qed: Photon-dependent qubit dephasing and relaxation rates. Phys. Rev. A 79, 013819 (2009).

23. Nigg, S. E. et al. Black-box superconducting circuit quantization. Phys. Rev. Lett. 108, 240502 (2012).

24. Heeres, R. W. et al. Cavity state manipulation using photon-number selective phase gates. Preprint at arXiv:1503.01496 (2015).

25. Wielinga, B. \& Milburn, G. Quantum tunneling in a kerr medium with parametric pumping. Phys. Rev. A 48, 2494 (1993).

26. Walls, D. F. \& Milburn, G. J. Quantum optics (Springer Science \& Business Media, 2007).

27. Meaney, C. H., Nha, H., Duty, T. \& Milburn, G. J. Quantum and classical nonlinear dynamics in a microwave cavity. EPJ Quantum Technol. 1, 1-23 (2014).

28. Minganti, F., Bartolo, N., Lolli, J., Casteels, W. \& Ciuti, C. Exact results for schrödinger cats in driven-dissipative systems and their feedback control. Sci. Rep. 6 (2016). 
29. Johansson, J., Nation, P. \& Nori, F. Qutip: an open-source python framework for the dynamics of open quantum systems. Comput. Phys. Commun. 183, 1760-1772 (2012).

30. Johansson, J., Nation, P. \& Nori, F. Qutip 2: a python framework for the dynamics of open quantum systems. Comput. Phys. Commun. 184, 1234-1240 (2013).

31. Bourassa, J., Beaudoin, F., Gambetta, J. M. \& Blais, A. Josephson-junctionembedded transmission-line resonators: from kerr medium to in-line transmon. Phys. Rev. A 86, 013814 (2012).

32. Demirplak, M. \& Rice, S. A. Adiabatic population transfer with control fields. J. Phys. Chem. A 107, 9937-9945 (2003).

33. Demirplak, M. \& Rice, S. A. Assisted adiabatic passage revisited. J. Phys. Chem. B. 109, 6838-6844 (2005).

34. Bason, M. G. et al. High-fidelity quantum driving. Nat. Phys. 8, 147-152 (2012).

35. Zhang, J. et al. Experimental implementation of assisted quantum adiabatic passage in a single spin. Phys. Rev. Lett. 110, 240501 (2013).

36. Khaneja, N., Reiss, T., Kehlet, C., Schulte-Herbruggen, T. \& Glaser, S. J. Optimal control of coupled spin dynamics: design of nmr pulse sequences by gradient ascent algorithms. J. Magn. Reson. 172, 296-305 (2005). http://www.sciencedirect. com/science/article/B6WJX-4DXT7T6-6/2/e86f276f798a97858c5a9df62d5f50c0.

37. Boutin, S., Andersen, C. K., Venkatraman, J., Ferris, A. J. \& Blais, A. Resonator reset in circuit qed by optimal control for large open quantum systems. Preprint at arXiv:1609.03170 (2016).

38. Goto, H. Bifurcation-based adiabatic quantum computation with a nonlinear oscillator network. Sci. Rep. 6, 21686 (2016).

39. Goto, H. Universal quantum computation with a nonlinear oscillator network. Phys. Rev. A 93, 050301 (2016).
40. Yamamoto, T. et al. Flux-driven josephson parametric amplifier. Appl. Phys. Lett 93, 042510 (2008). http://link.aip.org/link/?APL/93/042510/1.

41. Wustmann, W. \& Shumeiko, V. Parametric resonance in tunable superconducting cavities. Phys. Rev. B 87, 184501 (2013).

42. Krantz, P. et al. Single-shot read-out of a superconducting qubit using a josephson parametric oscillator. Nat. Commun. 7 (2016). doi:10.1038/ ncomms11417.

43. Kraus, B. et al. Preparation of entangled states by quantum markov processes. Phys. Rev. A 78, 042307 (2008).

cC) Open Access This article is licensed under a Creative Commons Attribution 4.0 International License, which permits use, sharing, adaptation, distribution and reproduction in any medium or format, as long as you give appropriate credit to the original author(s) and the source, provide a link to the Creative Commons license, and indicate if changes were made. The images or other third party material in this article are included in the article's Creative Commons license, unless indicated otherwise in a credit line to the material. If material is not included in the article's Creative Commons license and your intended use is not permitted by statutory regulation or exceeds the permitted use, you will need to obtain permission directly from the copyright holder. To view a copy of this license, visit http://creativecommons. org/licenses/by/4.0/.

(c) The Author(s) 2017

Supplementary Information accompanies the paper on the npj Quantum Information website (doi:10.1038/s41534-017-0019-1). 